\title{
Capacitor Type Biconical Antennas
}

\author{
Janis Galejs
}

\section{Contribution from Applied Research Laboratory, Sylvania Electronic Systems, a Division of Sylvania Electric Products Inc., 40 Sylvan Road, Waltham, Mass.}

(Received June 24, 1963; revised August 12, 1963)

\begin{abstract}
In this paper the biconical antenna analysis performed by C. T. Tai is extended to top-loaded and half-buried antenna structures which may have spherical cores of lossy dielectric. After computing the terminal admittance of the antenna, its input admittance is obtained by transmission line considerations. The numerical calculations emphasize antennas of dimensions which are small relative to the wavelength. Wide angle antennas of solid cones exhibit smaller radiation power factors (or products of available bandwidth and efficiency) than top-loaded antennas of small angle. The hemispherical antenna, located above a perfectly conducting ground plane, exhibits a larger radiation power factor than the corresponding half-buried antenna of the same total volume.
\end{abstract}

\section{Introduction}

Small capacitor type antennas have been discussed in literature by Wheeler [1947] and more recently by Galejs [1962]. This work has established relations between antenna impedance and such parameters as antenna volume or dielectric loading. A small cavity-backed annular slot has been compared with a top-loaded electric dipole by Galejs and Thompson [1962]. For a given antenna volume the top-loaded electric dipole exhibits a larger radiation power factor $p$ (or a larger product of bandwidth and efficiency). The cavity-backed annular slot may be approached by depressing the base of the top-loaded dipole below the ground level, which may be expected to gradually decrease $p$ of the dipole. However, the rate of this decrease has not been established and the analysis of a top-loaded dipole will be rather involved in a cylindrical geometry.

The effect of antenna burial may be considered more readily with biconical antennas. The theory of such antennas has been developed by Schelkunoff [1943] and Tai [1949]. The application of this theory to the analysis of a top-loaded biconical antenna, and to a half-buried antenna of figures 1 to 3 , is straightforward. It is possible to examine configurations with small $\theta_{0}$ and $\theta_{1}$ exceeding about $40^{\circ}$, or configurations with $\theta_{0}=\theta_{1}>40^{\circ}$. This analysis is tractable only if the boundaries between the regions follow a constant coordinate surface, which precludes the examination of intermediate burial depths. Still the configuration of figure 1 affords an interesting comparison with the cavity backed slot of Galejs and Thompson [1962]. The geometries of figures 1 to 3 will be considered by the same analysis technique, for several angles $\theta_{1}$ and various dielectric parameters of the antenna.

\section{Antenna Admittance}

The input admittance $Y_{i}$ of a biconical antenna of figure 1 is related to the effective terminating admittance $Y_{t}$ of the biconical transmission line [Schelkunoff, 1943] as

$$
Y_{i}=\frac{1}{K_{1}} \frac{K_{1} Y_{t} \cos k_{1} a+j \sin k_{1} a}{\cos k_{1} a+j K_{1} Y_{t} \sin k_{1} a}
$$

where

$$
\begin{gathered}
K_{1}=\frac{Z_{1}}{\pi} \log \cot \frac{\theta_{0}}{2} \\
Z_{i}=\sqrt{\frac{j \omega \mu_{0}}{\sigma_{i}+j \omega \epsilon_{i}}} \\
k_{i}=\sqrt{\omega^{2} \mu_{0} \epsilon_{i}-j \omega \mu_{0} \sigma_{i}} .
\end{gathered}
$$

The terminal admittance of the biconical antenna of figure 1 can be derived following the variational

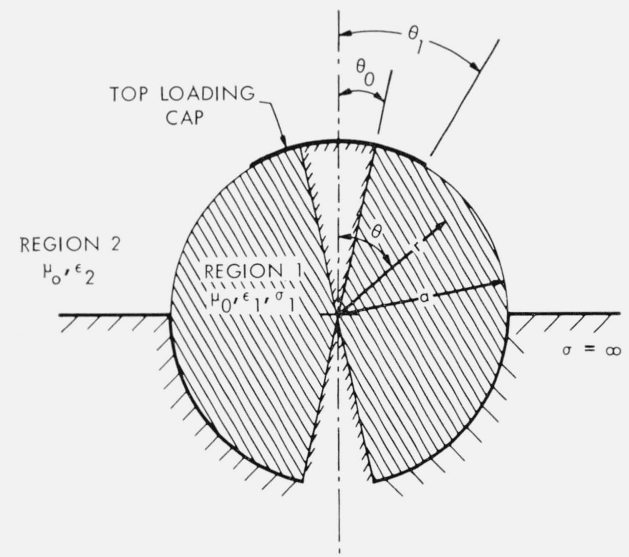

FigURE 1. The half-buried biconical antenna 


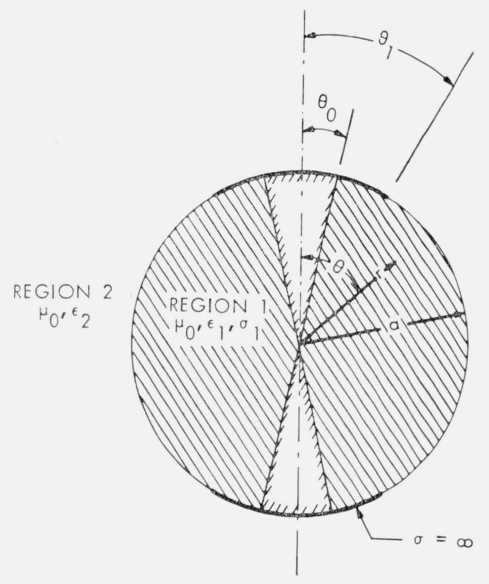

Figure 2. The symmetrical biconical antenna.

method of Tai [1949]. The terminal admittance $Y_{t}$ depends on integrals involving the electric field $E_{\theta}$ in the antenna aperture $r=a, \theta_{1}<\theta<\pi / 2$. The field $E_{\theta}(a, \theta)=E_{a}(\theta)$ is expressed as a series of Legendre functions. The coefficients of this series are determined from the stationarity property of the $Y_{t}$ expression. Using a two term approximation to $E_{a}(\theta), Y_{t}$ is of the form

$$
Y_{t}=Y_{t 0}-\frac{\alpha_{m 1}^{2}}{\beta_{m 1}}
$$

The term $Y_{t 0}$ is the zero order admittance which is due to the aperture field

$$
E_{a}(\theta)=-\frac{A_{0}}{\sin \theta} .
$$

The second term in (5) provides a correction to $Y_{t 0}$. It follows that

$$
\begin{aligned}
Y_{t 0}= & \frac{2 \pi j}{\left(\log \cot \frac{\theta_{1}}{2}\right)^{2}}\left\{\frac{1}{Z_{1}} \sum_{\nu} \frac{\left[L_{\nu}\left(\theta_{1}\right)\right]^{2}}{\nu(\nu+1) N_{\nu} I_{\nu \nu}}\right. \\
& \left.-\frac{1}{Z_{2}} \sum_{k} \frac{\left[P_{k}\left(\theta_{1}\right)\right]^{2}}{k(k+1) M_{k} I_{k k}}\right\},
\end{aligned}
$$

$\alpha_{m}=\frac{2 \pi j}{\left(\log \cot \frac{\theta_{1}}{2}\right)^{2}}\left\{\frac{1}{Z_{1}} \sum_{\nu} \frac{\left[L_{\nu}\left(\theta_{1}\right)\right] I_{\nu m}}{N_{\nu} I_{\nu \nu}}\right.$

$$
\left.-\frac{1}{Z_{2}} \sum_{k} \frac{P_{k}\left(\theta_{1}\right) I_{k m}}{M_{k} I_{k k}}\right\}
$$

$$
\begin{aligned}
\beta_{m}=\frac{2 \pi j}{\left(\log \cot \frac{\theta_{1}}{2}\right)^{2}}\left\{\frac{1}{Z_{2}} \sum_{\nu} \frac{\nu(\nu+1) I_{\nu m}^{2}}{N_{\nu} I_{\nu \nu}}\right. & \\
& \left.-\frac{1}{Z_{2}} \sum_{k} \frac{k(k+1) I_{k m}^{2}}{M_{k} I_{k k}}\right\} .
\end{aligned}
$$

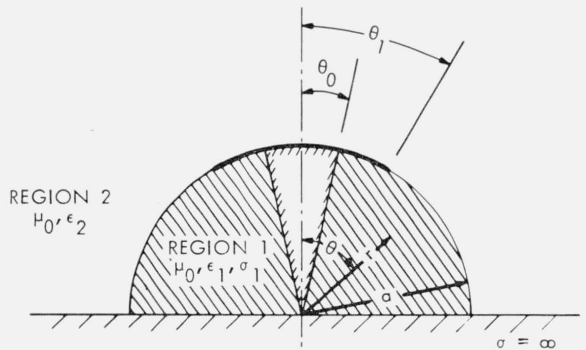

Figure 3. The top-loaded biconical antenna above an infinite ground plane.

In the above expressions

$$
\begin{gathered}
N_{\nu}=\frac{S_{\nu}^{\prime}\left(k_{1} a\right)}{S_{\nu}\left(k_{1} a\right)} \\
M_{k}=\frac{R_{k}^{\prime}\left(k_{2} a\right)}{R_{k}\left(k_{2} a\right)} \\
S_{\nu}(x)=\sqrt{x} J_{\nu+1 / 2}(x) \\
R_{k}(x)=\sqrt{x} H_{k+1 / 2}^{(2)}(x) .
\end{gathered}
$$

The prime denotes differentiation with respect to the argument of the respective function. $P_{\mu}(\theta)$ is a Legendre function of the first kind of order $\mu, J_{n}(x)$ is a Bessel function of order $n, H_{n}^{(2)}(x)$ is the Hankel function of the second kind of order $n$, and $k$ is restricted to odd integers. The Legendre function $L_{\nu}(\theta)$ is defined following Schelkunoff [1943] as

and

$$
L_{\nu}^{0}(\theta)=\frac{1}{2}\left[P_{\nu}(\theta)-P_{\nu}(\pi-\theta)\right]
$$

$$
L_{\nu}^{e}(\theta)=\frac{1}{2}\left[P_{\nu}(\theta)+P_{\nu}(\pi-\theta)\right]
$$

where $\nu$ is such that $L_{\nu}(\theta)=0$ for $\theta=\theta_{0}$ and $\left(\pi-\theta_{0}\right)$. $L_{\nu}(\theta)$ defined by (14) is an odd function of $\cos \theta$, while (15) is an even function.

The symbols $I_{i j}$ of (7) to (9) involve integrals of Legendre functions. Thus

$$
I_{k k}=\int_{0}^{\pi / 2}\left[P_{k}(\theta)\right]^{2} \sin \theta d \theta=\frac{1}{2 k+1}
$$

$\theta_{1}$ is selected such that $P_{m}\left(\theta_{1}\right)=P_{m}\left(\pi-\theta_{1}\right)=0$ for $m$ equal to an odd integer. Then

$$
\begin{gathered}
I_{k m}=\int_{\theta 1}^{\pi / 2} P_{k}(\theta) P_{m}(\theta) \sin \theta d \theta \\
=-\frac{m}{k^{2}+k-m^{2}-m} P_{k}\left(\theta_{1}\right) P_{m-1}\left(\theta_{1}\right) \quad(\text { if } k \neq m),
\end{gathered}
$$




$$
\begin{aligned}
& I_{m m}=\int_{\theta_{1}}^{\pi / 2}\left[P_{m}(\theta)\right]^{2} \sin \theta d \theta \\
&=\frac{2}{2 m+1}\left[\frac{P_{0}\left(\theta_{1}\right) P_{1}\left(\theta_{1}\right)}{1.3}+\frac{P_{1}\left(\theta_{1}\right) P_{2}\left(\theta_{1}\right)}{3.5}+\cdots\right. \\
& \\
&\left.\quad+\frac{P_{m-2}\left(\theta_{1}\right) P_{m-1}\left(\theta_{1}\right)}{(2 m-3)(2 m-1)}\right] .
\end{aligned}
$$

For $\theta_{0}<<1, L_{\nu}(\theta)$ of (14) or (15) is approximated following Schelkunoff [1943] by

$$
L_{\nu}(\theta)=P_{\nu}(\theta)-\delta Q_{\nu}(\theta)
$$

where

$$
\begin{gathered}
\nu=n+\delta \\
\delta=\left[\log \frac{2}{\theta_{0}}\right]^{-1}
\end{gathered}
$$

$n$ is an integer or zero and where $Q_{\nu}(\theta)$ is a Legendre function of the second kind of order $\nu$. Then $I_{\nu m}$ is given by

$$
\begin{aligned}
I_{\nu m} & =\int_{\theta_{1}}^{\pi / 2} L_{\nu}(\theta) P_{m}(\theta) \sin \theta d \theta \\
& =\left.\frac{m}{\nu^{2}+\nu-m^{2}-m} P_{m-1}(\theta) L_{\nu}(\theta)\right|_{\theta_{1}} ^{\pi / 2}
\end{aligned}
$$

where $\nu \neq m$. The integral $I_{\nu \nu}$ is obtained as

$$
I_{\nu \nu}=\operatorname{Lim}_{\Delta \rightarrow 0} \int_{\theta_{0}}^{\pi-\theta_{0}} L_{\nu}(\theta) L_{\nu+\Delta}(\theta) \sin \theta d \theta .
$$

Substituting (19), and using the approximation of $P_{\nu}(\theta)$ and $Q_{\nu}(\theta)$ for the vicinity of $\theta=0$ and $\pi$ it follows that

$$
\begin{aligned}
I_{\nu \nu}= & \frac{2}{2 n+1}\left\{1-\delta \frac{2}{2 n+1}+\delta^{2}\right. \\
& {\left.\left[\frac{\pi^{2}}{4}-\sum_{r=0}^{\infty}(1+n+r)^{-2}+\left(\frac{2}{2 n+1}\right)\right]^{2}+\ldots\right\} }
\end{aligned}
$$

where terms proportional to higher powers of $\delta$ and proportional to $\theta_{0}^{2}$ have been neglected.

For $\theta_{0}=\theta_{1}$ the terms of (7) and (8) which are proportional to $L_{\nu}\left(\theta_{1}\right)$ are equal to zero. The part of the summation over $\nu$ in (9) which contains integrals of the odd functions $L_{\nu}^{0}(\theta)$ is reduced to a single term with $I_{\nu m}=I_{m m}$ and $I_{\nu \nu}=2 I_{m m}$. The integrals in $\Sigma_{\nu}$ of (9) which involve the even functions $L_{\nu}^{e}(\theta)$ are denoted by superscripts as $I_{v m}^{e}$ and $I_{\nu \nu}^{e}$. For $\theta_{0}=\theta_{1}$ (7) to (9) are changed into

$$
Y_{t 0}=-\frac{2 \pi j}{\left(\log \cot \frac{\theta_{1}}{2}\right)^{2}} \frac{1}{Z_{2}} \sum_{k} \frac{\left[P_{k}\left(\theta_{1}\right)\right]^{2}}{k(k+1) M_{k} I_{k k}}
$$

$$
\begin{gathered}
\alpha_{m}=-\frac{2 \pi j}{\left(\log \cot \frac{\theta_{1}}{2}\right)^{2}} \frac{1}{Z_{2}} \sum_{k} \frac{P_{k}\left(\theta_{1}\right) I_{k m}}{M_{k} I_{k k}} \\
\beta_{m}=\frac{2 \pi j}{\left(\log \cot \frac{\theta_{1}}{2}\right)^{2}}\left\{\frac{1}{Z_{1}} \frac{m(m+1) I_{m m}}{2 N_{m}}\right. \\
\left.+\frac{1}{Z_{1}} \sum_{\nu} \frac{\nu(\nu+1) I_{\nu m}^{e^{2}}}{N_{\nu} I_{\nu \nu}^{e}}-\frac{1}{Z_{2}} \sum_{k} \frac{k(k+1) I_{k m}^{2}}{M_{k} I_{k k}}\right\}
\end{gathered}
$$

with the integrals $I_{k k}, I_{k m}$, and $I_{m m}$ defined by (16), (17), and (18) respectively. After substituting (15) in (22) $I_{\nu m}^{e}$ is obtained as

$$
I_{\nu m}^{e}=-\frac{2 m}{\nu^{2}+\nu-m^{2}-m} P_{m-1}\left(\frac{\pi}{2}\right) P_{\nu}\left(\frac{\pi}{2}\right) .
$$

Substituting (15) in (23) it follows that

$$
\begin{aligned}
I_{\nu \nu}^{e} & =-\left.\frac{\sin \theta}{2 \nu+1} \frac{\partial L_{\nu}^{e}\left(\theta_{1}\right)}{\partial \nu} \frac{\partial L_{\nu}^{e}(\theta)}{\partial \theta}\right|_{\theta_{1}} ^{\pi-\theta_{1}} \\
& =\frac{\nu}{2 \nu+1} \frac{\partial L_{\nu}^{e}\left(\theta_{1}\right)}{\partial \nu}\left[P_{\nu}\left(\theta_{1}\right)-P_{\nu}\left(\pi-\theta_{1}\right)\right]
\end{aligned}
$$

The characteristic values $\nu=\nu_{i}$ which result in $L_{\nu}^{e}\left(\theta_{1}\right)=0$ and also the derivatives $\partial L_{\nu}^{e}\left(\theta_{1}\right) / \partial \nu$ for $\nu=\nu_{i}$ are obtained by numerically computing $L_{\nu}^{e}\left(\theta_{-}\right)$ as a function of $\nu$. The zeros $\nu_{i}$ can be also obtained by interpolation from the zeros of $P_{2 n}(\theta)$ and $Q_{2 n+1}(\theta)$, where $n$ is an integer.

The input admittance $Y_{i}$ of a symmetrical biconical antenna of figure 2 is related to the terminal admittance $Y_{t}$ by (1). $\quad Y_{t}$ is given by (5) together with (7) to (9) or (25) to (27). The fields are symmetrical with respect to the $\theta=\pi / 2$ plane and only the odd functions $L_{\nu}^{0}(\theta)$ should be considered. For $\theta_{0}<<\theta_{1}, \nu=k+\delta$ where $k$ is an odd integer. For $\theta_{0}=\theta_{1}$, the $\Sigma_{\nu}$ of (27) in the $\beta_{m}$ expression is equal to zero. However, the range of integration of the integrals $I_{k k}, I_{k m}, I_{m m}$, and $I_{\nu m}$ is doubled, which doubles their values relative to those indicated in (16), (17), (18), and (22). For $\theta_{0}=\theta_{1}$ and $\sigma_{1}=0$ this terminal admittance is the same as $Y_{t}$ in (68) of Galejs [1962].

The input admittance $Y_{i}$ of a biconical antenna above an infinite ground plane in figure 3 is two times $Y_{i}$ of the corresponding symmetrical biconical antenna in figure 2.

\section{Elementary Design Considerations}

The half-buried antenna structure of figure 1 is compared in this note with an antenna which has the same above-the-ground structure (fig. 3) (called antennas 1 and 3 ). It can also be shown by elementary considerations that antenna 1 is superior to antenna 3 . However, antenna 3 becomes the better one, if its volume is increased to be equal to the total volume of antenna 1 . 


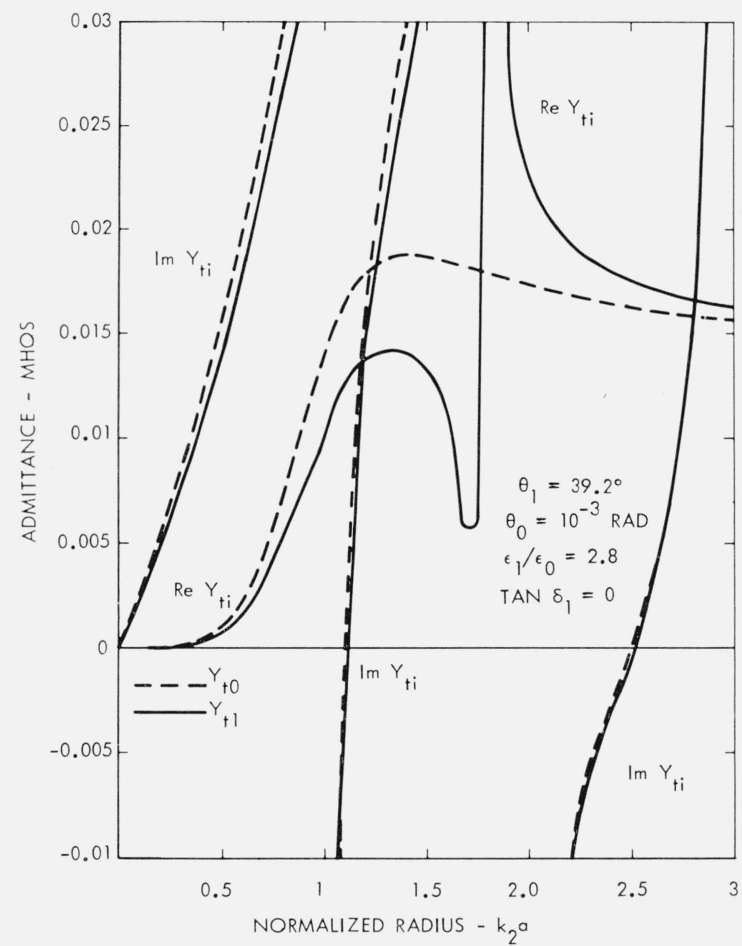

Figure 4. Terminal admittance of a half-buried cap-loaded biconical antenna (case 1a) loss-less dielectric.

Antennas 1 and 3 are compared for constant voltage $V$ across the aperture. These antennas have approximately the same magnetic dipole moment. The radiated power $P$ and the radiation conductance $G_{r}=P_{r} / V^{2}$ are therefore approximately constant for antennas 1 and 3 . Geometrical considerations show that the antenna capacities are related as

$$
C_{3}>C_{1}>C_{3} / 2
$$

The radiation power factors $p=G_{r} / \omega C$ are related for $G_{r}=$ constant as $p_{1} / p_{3}=C_{3} / C_{1}$. Applying the inequality (30) gives

$$
p_{3}<p_{1}<2 p_{3} \text {. }
$$

The loss conductance $G_{L}$ of the antenna due to dielectric losses may be defined from $P_{L}=\sigma \int E^{2} d v=$ $(\sigma / \epsilon) C_{i} V^{2}$, where $C_{i}$ is the capacity of the antenna associated with the fields inside the dielectric (the fringing fields are excluded from the definition of $C_{i}$; hence, $\left.C_{i}<C\right)$. It follows that $G_{L}=\sigma C_{i} / \epsilon$. With $G_{r}=$ constant, $\quad$ eff $1 / \mathrm{eff}_{3}=G_{L 3} / G_{L 1}=C_{i 3} / C_{i 1}$. The inequality (30) applies also to $C_{i}$. Hence

$$
2 \mathrm{eff}_{3}>\mathrm{eff}_{1}>\mathrm{eff}_{3} .
$$

The antenna structure 4 is obtained by increasing: the linear dimensions of antenna 3 by $2^{1 / 3} \approx 1.26$. (The volume of antenna 4 is the same as the total volume of antenna 1.) Hence, $C_{4}=2^{1 / 3} C_{3}, p_{4}$ $=2 p_{3}, \quad G_{L 4}=2^{1 / 3} G_{L 3}, \quad G_{r 4}=2^{4 / 3} G_{r 3}$, and $\mathrm{eff}_{4}=2 \quad \mathrm{eff}_{3}$.

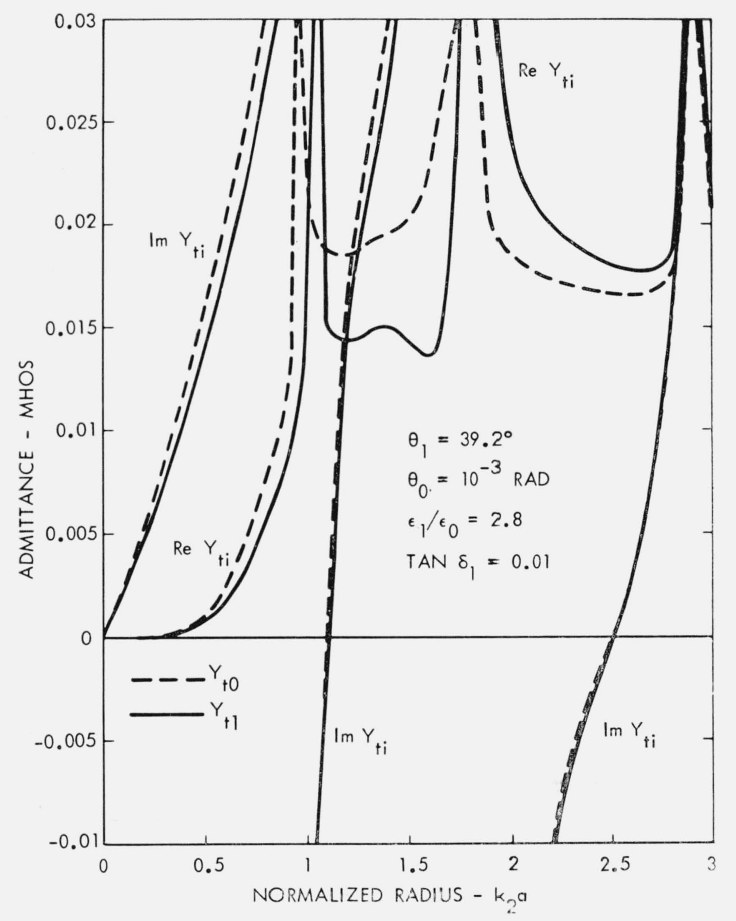

Figure 5. Terminal admittance of a half-buried cap-loaded biconical antenna (case 1a) lossy dielectric.

Comparison of antennas 1 and 4 yields

$$
\begin{gathered}
p_{4} / 2<p_{1}<p_{4} \\
\mathrm{eff}_{4} / 2<\mathrm{eff}_{1}<\mathrm{eff}_{4} .
\end{gathered}
$$

For a given volume the above-ground antenna 4 is superior to the half-buried antenna 1. The calculations or measurements which specify the input admittance

$$
Y_{i}=\left(G_{L}+G_{r}\right)+j \omega C \approx G_{L}+j \omega C
$$

are sufficient for specifying the ratios between radiation power factors $p$ or efficiencies eff of the antenna types under consideration. It follows from the preceding development that

$$
\begin{gathered}
\frac{p_{4}}{p_{1}}=\frac{2 p_{3}}{p_{1}}=\frac{2 C_{1}}{C_{3}} \\
\frac{\mathrm{eff}_{4}}{\mathrm{eff}_{1}}=\frac{2 \mathrm{eff}_{3}}{\mathrm{eff}_{1}}=\frac{2 G_{L 1}}{G_{L 3}} .
\end{gathered}
$$

\section{Discussion of Numerical Results}

Numerical results will be presented for halfburied antennas of figure 1 and above-the-ground antennas of figure 3 , which will be designated in the subsequent discussion as cases 1 and 3 , respectively. The configurations involving top loading spherical caps $\left(\theta_{0}<<\theta_{1}\right)$ and solid wide angle cones 


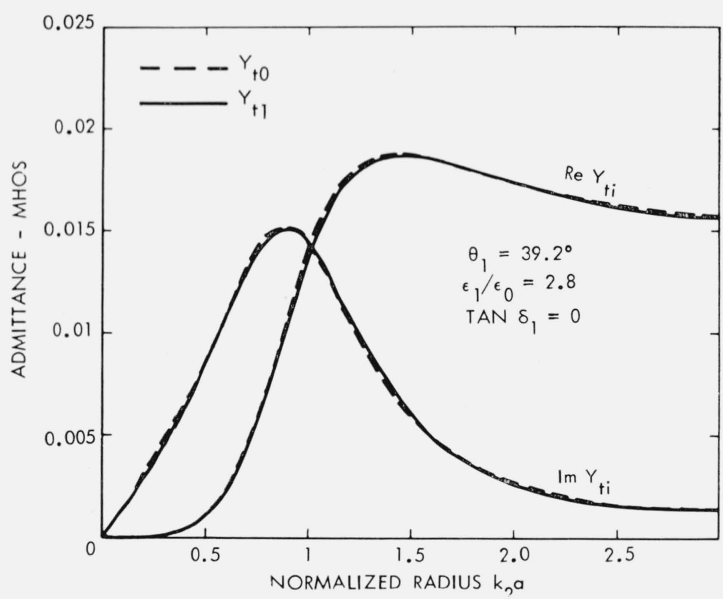

Figure 6. Terminal admittance of a half-buried wide angle biconical antenna (case 1b).

$\left(\theta_{0}=\theta_{1}\right)$ are labeled by subscripts $a$ and $b$ respectively.

The zero-order terminal admittance $Y_{t 0}$, is compared with the first-order terminal admittance $Y_{t 1}$ in figures 4,5 , and 6 for cases $1 \mathrm{a}$ and $1 \mathrm{~b}$. There are no appreciable differences between $Y_{t 0}$ and $Y_{t 1}$ except for the difference between $\operatorname{Re} Y_{t 0}$ and $\operatorname{Re} Y_{t 1}$ near the resonance of case 1a in figure 4 . This difference is decreased for a lossy dielectric in figure 5. There are no such resonance phenomena for case $1 \mathrm{~b}$. The terminal admittance for case $1 \mathrm{~b}$ in figure 6 is twice the free-space terminal admittance of the wide-angle biconical antenna of Tai [1949].

The input admittance $Y_{i}$ is related by the transmission line equation (1) to the terminal admittance $Y_{t}$. The calculated values of $B_{i}=\operatorname{Im} Y_{i}, G_{i}=\operatorname{Re} Y_{i}$ $=G_{r}$ for $\tan \delta=0, G_{i}=\operatorname{Re} Y_{i}=G_{r}+G_{L}$ for $\tan \delta_{1}=0.01$ are plotted in figures 7 to 9 . There are additional transmission line resonances in figures 7 to 9 . For increased terminal capacities of the biconical transmission line $\left(\theta_{1}\right.$ large) the imaginary part of the denominator in (1) becomes zero at smaller values of $k_{2} a$. The first resonance of figure $8\left(\theta_{1}=66^{\circ}\right)$ occurs therefore at a smaller $k_{2} a$ value than in figure $7\left(\theta_{1}=39.2^{\circ}\right)$. The differences between the input admittance plots of case $1 \mathrm{a}$ in figures 7 and 8 and case $1 \mathrm{~b}$ in figure 9 are due to the differences between the terminal admittances in figures 4 or 5 and figure 6 .

The characteristics of small biconical antennas $\left(k_{2} a<0.2\right)$ are examined more closely in figures 10 to 17 .

The radiation conductance $G_{r}$ is plotted in figures 10 and 11 . For antennas of $k_{2} a<0.05, G_{r}$ is proportional to $\left(k_{2} a\right)^{4}$. Over this range $G_{r}$ is essentially independent of the internal antenna structure and of the dielectric loading. Larger differences between the various antenna types occur for larger values of $\theta_{1}$ when the transmission line resonance occurs at smaller values of $k_{2} a$, as may be seen from a comparison of figures 7 and 8 .

The antenna susceptance $B=\operatorname{Im} \quad Y_{i}$ has been plotted in figures 12 to 14 for $\epsilon_{1} / \epsilon_{0}=1$ and 2.8. The

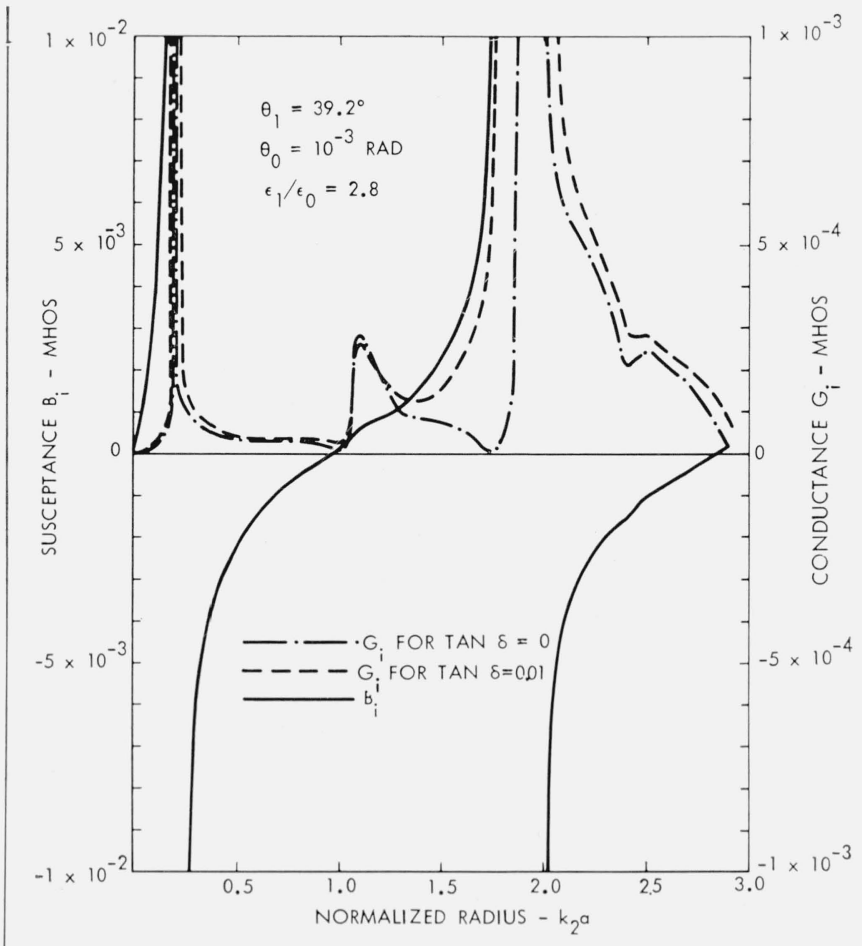

FIGURE 7. Input admittance of a half-buried cap-loaded biconical antenna (case 1a) $\theta_{1}=39.2^{\circ}$.

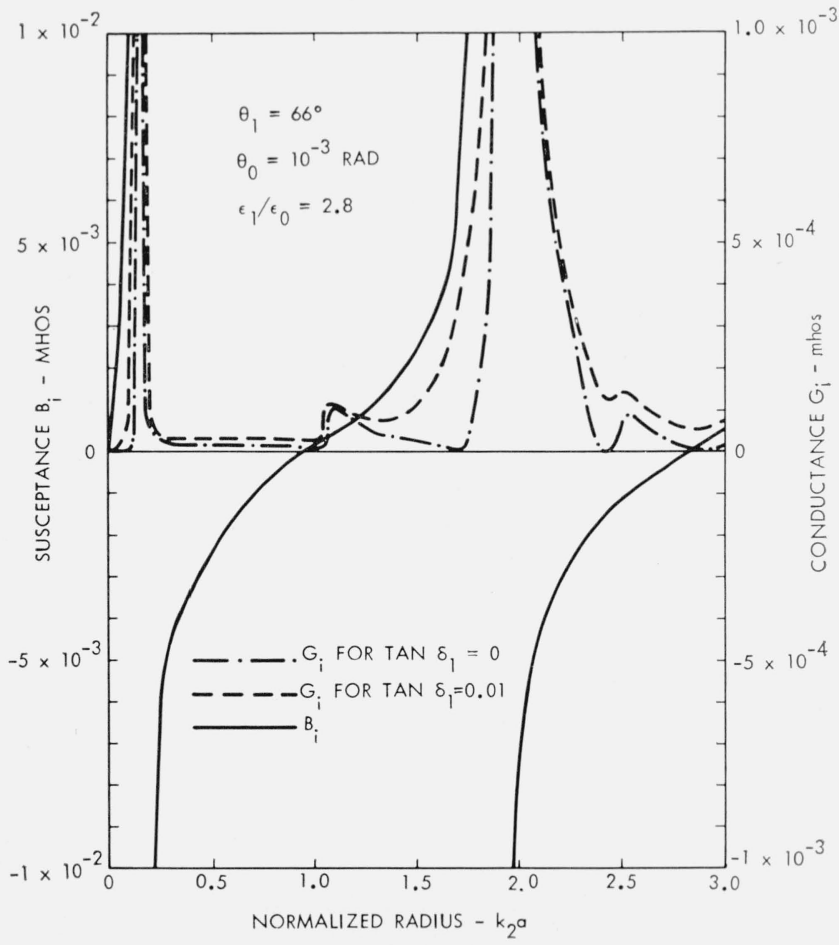

Figure 8. - Input admittance of a half-buried cap-loaded biconical antenna (case 1a) $\theta_{1}=66^{\circ}$. 


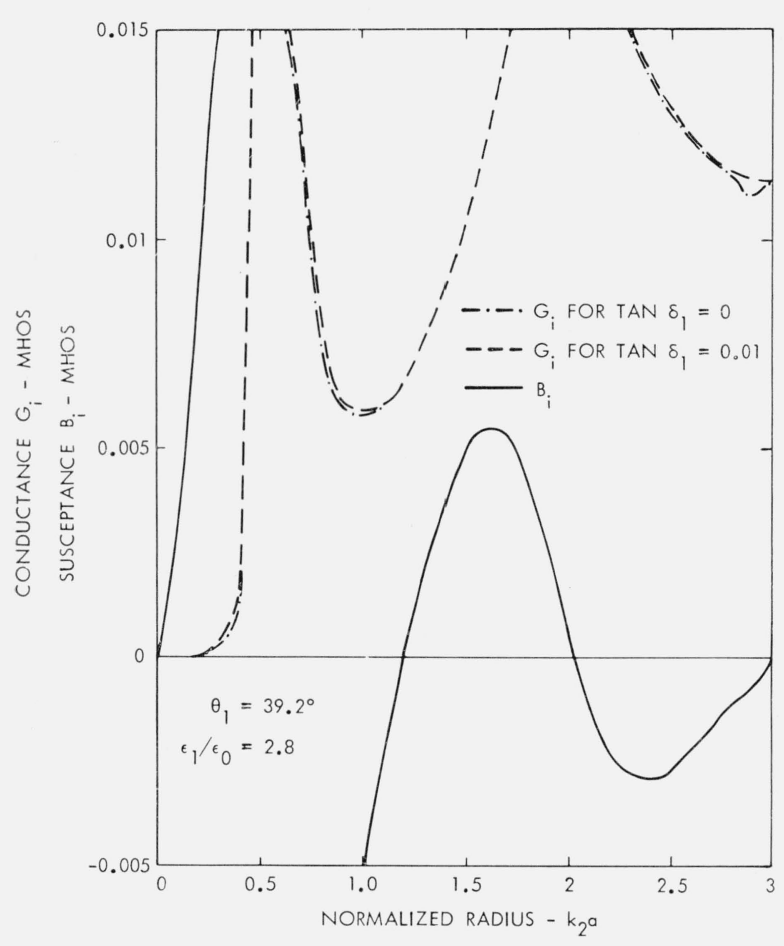

FIGURE 9. Input admittance of a half-buried wide-angle biconical antenna (case $1 b) \theta_{1}=39.2^{\circ}$.

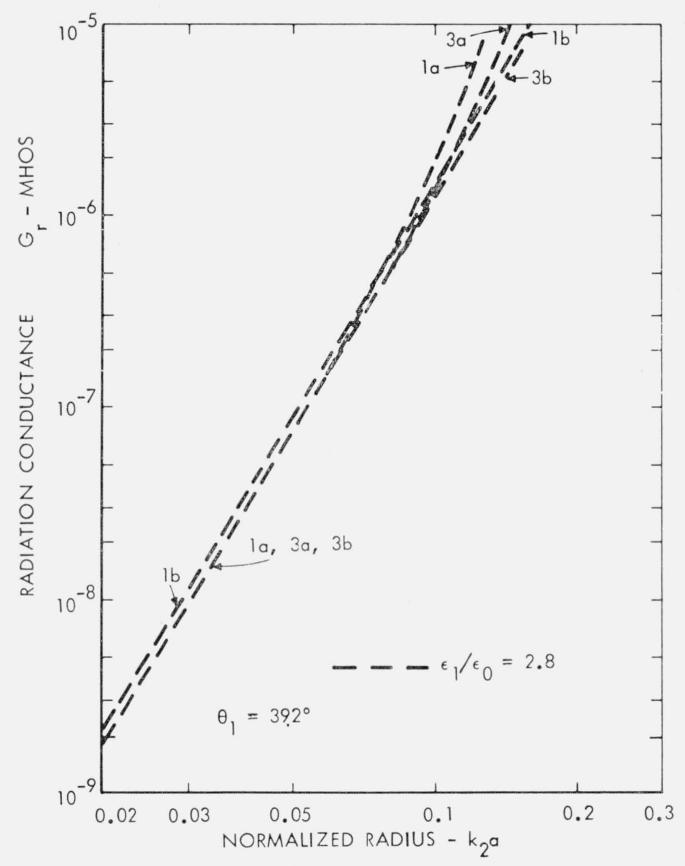

Figure 10. Radiation conductance of cap-loaded and wideangle biconical antennas (cases $1 a, 1 b, 3 a$, and $3 b)$.

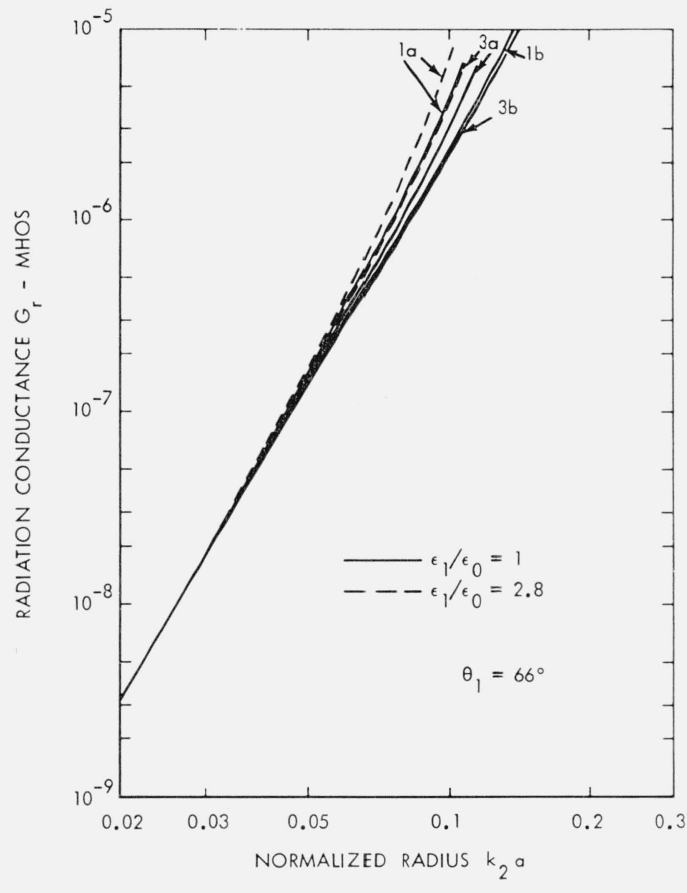

FIgURE 11. Radiation conductance of cap-loaded and wideangle biconical antennas (cases 1a, 1b, 3a, and $3 b$ ).

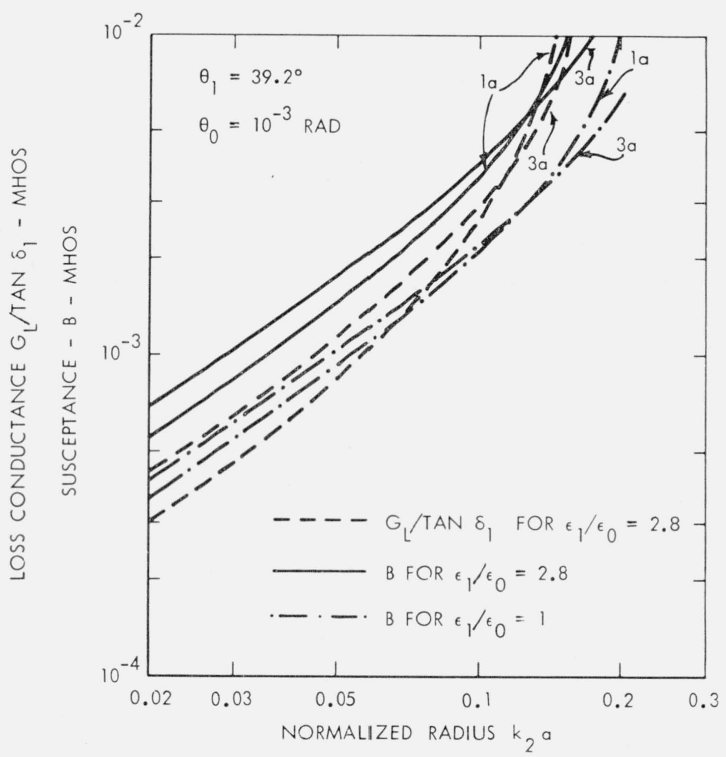

Figure 12. Loss conductance and susceptance of cap-loaded biconical antennas (cases $1 a$ and $3 a$ ) $\theta_{1}=39.2^{\circ}$. 


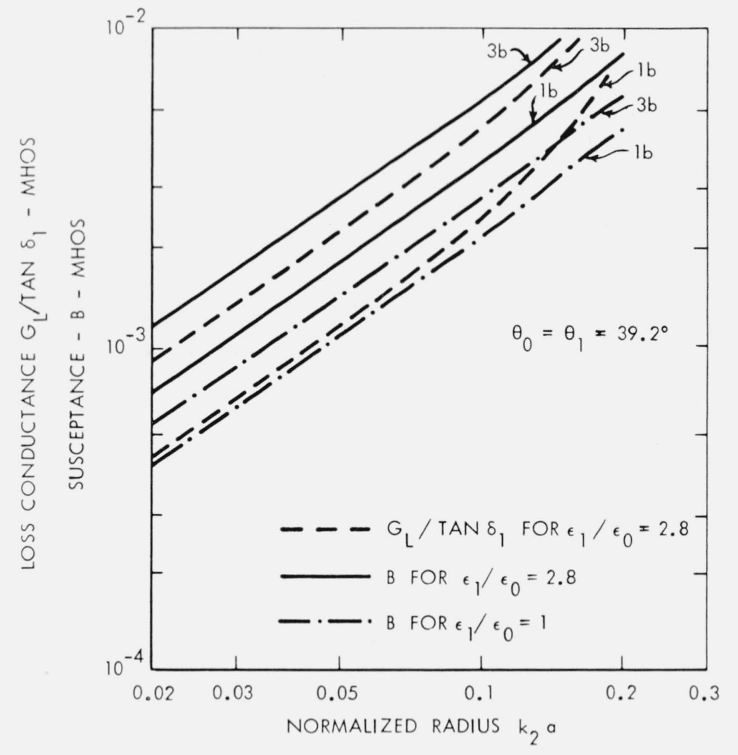

FIgURE 13. Loss conductance and susceptance of wide-angle biconical antennas (cases $1 b$ and 36 ) $\theta_{1}=39.2^{\circ}$.

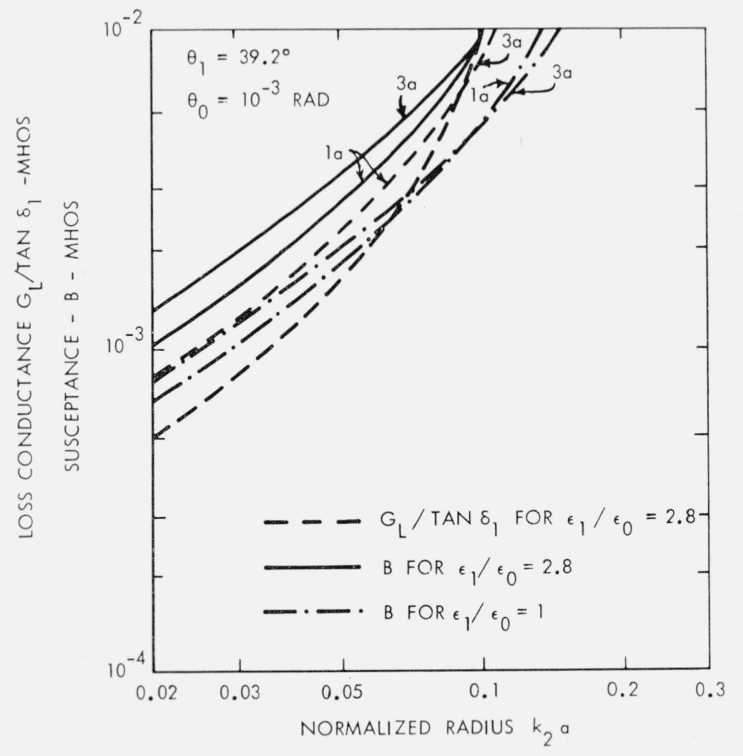

Figure 14. Loss conduclance and susceptance of cap-loaded biconical antennas (cases $1 a$ and 8 a) $\theta_{1}=66^{\circ}$.

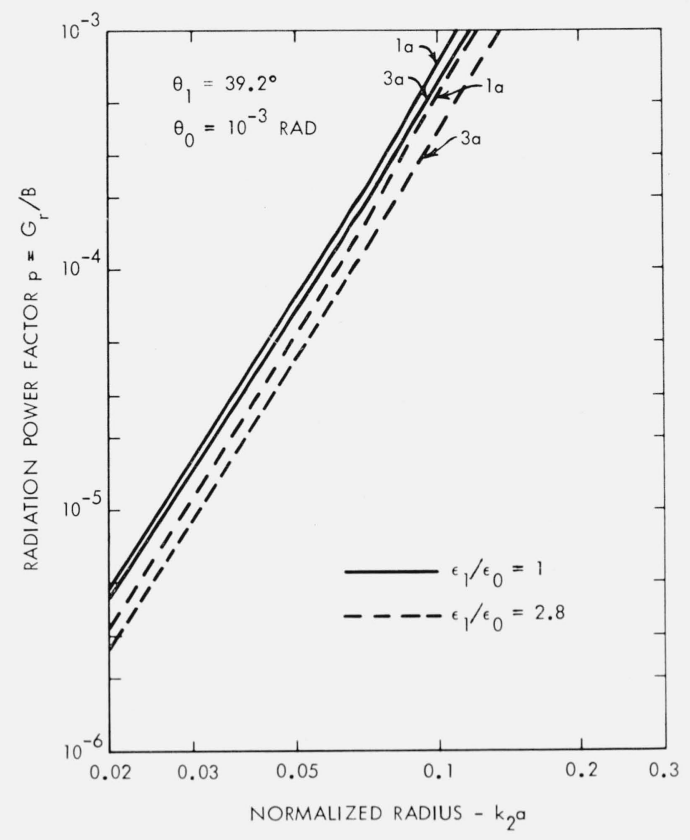

Figure 15. Radiation power factors of cap-loaded biconical antennas (cases $1 a$ and $3 a$ ) $\theta_{1}=39.2^{\circ}$.

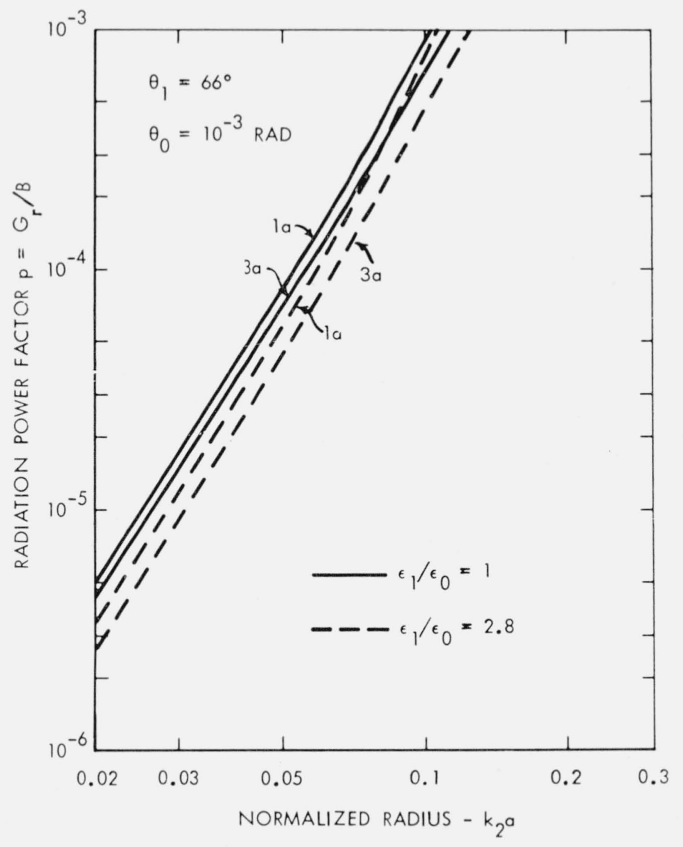

Figure 16. Radiation power factors of cap-loaded biconical antennas (cases $1 a$ and $3 a$ ) $\theta_{1}=66^{\circ}$. 


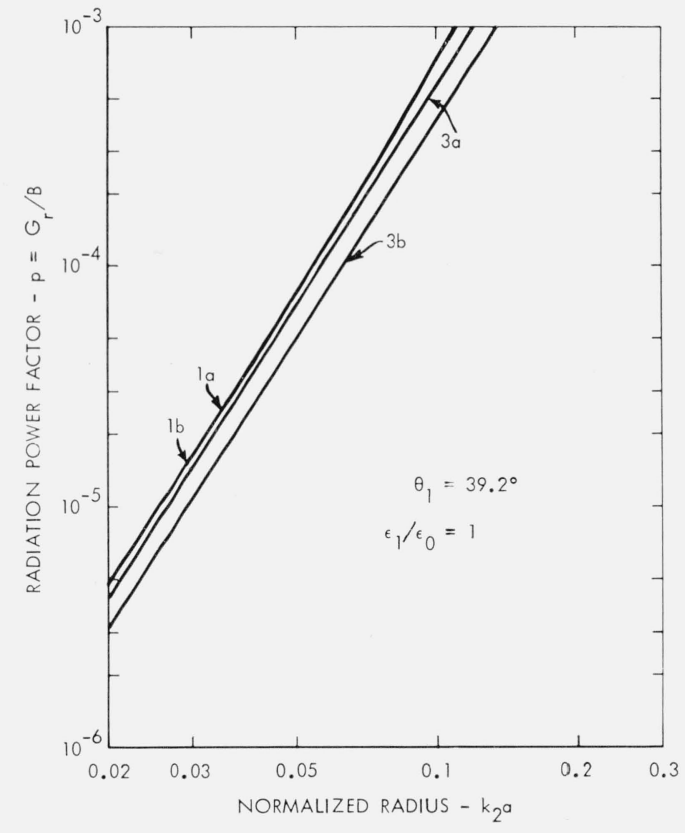

FIgure 17. Radiation power factors cap-loaded and wide-angle biconical antennas (cases $1 a, 1 b, 3 a$, and $3 b$ ) $\theta_{1}=39.2^{\circ}$.

same figures contain also plots $G_{L} / \tan \delta$ for $\epsilon_{1} / \epsilon_{0}=2.8$ which is equal to the susceptance $B_{i}=\omega C_{i}$ associated with fields inside the dielectric. $B$ and $G_{L}$ are proportional to $k_{2} a$ for $k_{2} a<<1$. In the configuration of case 3 there is less fringing of the fields, and $G_{L} /$ $\tan \delta$ differs less from $B$ than in case 1. However, $G_{L} / \tan \delta_{1}$ may exceed $|B|$ for values of $k_{2} a$ near the resonance of the transmission line. The resonance is approached for $k_{2} a>0.1$.

The plots of figures 10 to 14 provide the data for computing the radiation efficiency

$$
\mathrm{eff}=\frac{G_{r}}{G_{L}+G_{r}} \approx \frac{G_{r}}{G_{L}}
$$

the relative antenna bandwidth

$$
\mathrm{BW}=\frac{G_{L}+G_{r}}{B} \approx \frac{G_{L}}{B}
$$

or the radiation power factor

$$
p=(\mathrm{BW}) \cdot(\text { eff })=\frac{G_{r}}{B} .
$$

Calculated values of the radiation power factor $p$ are shown in figures 15 to 17 . The power factors $p$ are proportional to $\left(k_{2} a\right)^{3}$. The power factors $p$ are largest for $\epsilon_{1} / \epsilon_{0}=1 ; p_{a}$ for $\theta_{0}<<\theta_{1}$ (configuration $a$ ) exceeds $p_{b}$ for $\theta_{0}=\theta_{1}$ (configuration $b$ ) because of $C_{a}<C_{b}$ for $G_{a} \approx G_{b}$. The power factor $p_{1}$ of case 1 exceeds $p_{3}$ of case 3 because of larger total antenna volume for a given value of $k_{2} a$, as pointed out in section 3. However, the antenna of case 1 exhibits the smaller power factor if the volume of the antenna of case 3 is made equal in volume to the antenna of case 1 . This is achieved by increasing $\left(k_{2} a\right)$ of case 3 by a factor of $2^{1 / 3} \approx 1.26$. The above argument will be illustrated with an example for $\theta_{1}=39.2^{\circ}$ and $k_{2} a=0.05$. The calculations of eff, $\mathrm{BW}$, and $p$ will be made in the cases of $1 \mathrm{a}, 1 \mathrm{~b}, 3 \mathrm{a}, 3 \mathrm{~b}$ and for antennas of case 3 with a doubled volume (cases $4 \mathrm{a}$ and $4 \mathrm{~b}$ ). The results listed in table 1 are also in accord with the discussion of section 3 .

TABle 1. Power factors, bandwidth, and efficiency figures above-the-ground and half-buried antennas

$k_{2} a=0.05, \quad \theta_{1}=39.2^{\circ}, \quad \epsilon_{1} / \epsilon_{0}=2.8, \quad \tan \delta_{1}=0.01$.

\begin{tabular}{c|c|c|c}
\hline \hline Configurations & $p$ & BW & eff \\
\hline Case $1 \mathrm{a}$ & $5.25 \times 10^{-5}$ & $5.8 \times 10^{-3}$ & $9.1 \times 10^{-3}$ \\
$3 \mathrm{a}$ & $4.22 \times 10^{-5}$ & $6.5 \times 10^{-3}$ & $6.5 \times 10^{-3}$ \\
$4 \mathrm{a}$ & $8.44 \times 10^{-5}$ & $6.5 \times 10^{-3}$ & $13 \times 10^{-3}$ \\
\hline Case 1b & $4.69 \times 10^{-5}$ & $6.3 \times 10^{-3}$ & $7.5 \times 10^{-3}$ \\
$3 \mathrm{~b}$ & $2.6 \times 10^{-5}$ & $7.8 \times 10^{-3}$ & $3.33 \times 10^{-3}$ \\
$4 \mathrm{~b}$ & $5.2 \times 10^{-5}$ & $7.8 \times 10^{-3}$ & $6.66 \times 10^{-3}$ \\
\hline
\end{tabular}

Appreciation is expressed to T. W. Thompson for checking the derivations and to Miss E. Marley for computational assistance.

\section{References}

Galejs, J. (Sept.-Oct. 1962), Dielectric loading of electric dipole antennas, J. Res. NBS 66D (Radio Prop.), No. 5, $557-562$.

Galejs, J., and T. W. Thompson (Nov. 1962), Admittance of a cavity-backed annular slot antenna, IRE Trans. Ant. Prop. AP-10, 671-678.

Schelkunoff, S. A. (1943), Electromagnetic Waves (D. Van Nostrand Co., New York, N.Y.).

Tai, C. T. (Nov. 1949), Application of a variational principle of biconical antennas, J. App. Phys. 20, No. 11, 1076-1084.

Wheeler, H. A. (Dec. 1947), Fundamental limitations of small antennas, Proc. IRE 35, No. 12, 1479-1484.

(Paper 68D2-329) 